\title{
Alcohol and Vapourized Nicotine Co-Exposure During Adolescence Contribute Differentially to Sex-Specific Behavioural Effects in Adulthood
}

\author{
Jessica Ruffolo ${ }^{1,2^{*}}$, Jude A. Frie ${ }^{1,2^{*}}$, Hayley H.A. Thorpe ${ }^{1,2}$, \\ M. Asfandyaar Talhat ${ }^{1}$, Jibran Y. Khokhar ${ }^{1,2}$
}

${ }^{1}$ Department of Biomedical Sciences, University of Guelph, Guelph, ON, Canada

${ }^{2}$ Collaborative Neurosciences Graduate Program, University of Guelph, Guelph, ON, Canada

* Both authors contributed equally to this work

Corresponding Author:

Jibran Y. Khokhar, Ph.D.

jkhokhar@uoguelph.ca 


\section{ABSTRACT}

Introduction: Co-occurrence of e-cigarette use and alcohol consumption during adolescence is frequent. However, little is known about their long-lasting effects when combined. Here, we examined whether adolescent co-exposure to alcohol drinking and vapourized nicotine would impact reward- and cognition-related behaviours in adult male and female rats during adulthood.

Methods: Four groups of male and female Sprague Dawley rats ( $n=8-11 /$ group/sex) received either nicotine (JUUL $5 \%$ nicotine pods) or vehicle vapour daily between postnatal days 30-46, while having continuous voluntary access to ethanol and water during this time in a two-bottle preference design. Upon reaching adulthood, rats underwent behavioural testing utilizing Pavlovian conditioned approach testing, fear conditioning and a two-bottle alcohol preference test.

Results: A sex-dependent effect was found in the two-bottle preference test in adulthood such that females had a higher intake and preference for alcohol compared to males regardless of adolescent exposure; both male and female adult rats had greater alcohol preference compared to adolescents. Male rats exposed to vapourized nicotine with or without alcohol drinking during adolescence exhibited altered rewardrelated learning in adulthood, evidenced by enhanced levels of sign-tracking behaviour. Male rats that drank alcohol with or without nicotine vapour in adolescence showed deficits in associative fear learning and memory as adults. In contrast, these effects were not seen in female rats exposed to alcohol and nicotine vapour during adolescence. 
Conclusions: The present study provides evidence that co-exposure to alcohol and vapourized nicotine during adolescence in male, but not female, rats produces longterm changes in reward- and cognition-related behaviours.

Implications: These findings enhance our understanding of the effects of alcohol drinking and nicotine vapour exposure in adolescence. Moreover, they highlight potential sex differences that exist in the response to alcohol and nicotine vapour, underscoring the need for follow-up studies elucidating the neurobiological mechanisms that drive these sex differences, as well as the long-term effects of alcohol and nicotine vapour use. 


\section{INTRODUCTION}

E-cigarettes have become increasingly popular among adolescents ${ }^{1}$; in $2020,30.7 \%$ of $10^{\text {th }}$ graders and $34.5 \%$ of $12^{\text {th }}$ graders reported using e-cigarettes, following a two-fold increase over the past two years ${ }^{1}$. An equally important issue is the consumption of alcohol by adolescents. Over $78 \%$ of adolescents have consumed alcohol by late adolescence, and 15\% meet the criteria for DSM-IV lifetime alcohol abuse ${ }^{2}$. Alcohol and nicotine are frequently used sequentially and simultaneously by adolescents ${ }^{3}$ with a high prevalence of concurrent e-cigarette vaping and alcohol consumption ${ }^{4}$. Vaping high school students also showed greater alcohol drinking compared to non-vapers ${ }^{5}$, and alcohol and e-cigarette use is the most common type of co-use in this population ${ }^{6}$. The long-term effects of vaping are currently unknown, as are the consequences of ecigarette/alcohol co-use ${ }^{6}$. In contrast, the additive effects of combustible tobacco and alcohol co-use are well established. For example, cigarette smoking amplifies cognitive deficits in adults who excessively drink alcohol, and alcohol-dependent adults who smoke cigarettes show pronounced neuropsychological damage compared to alcoholdependent non-smokers ${ }^{7,8}$. Unfortunately, the consequences of nicotine and alcohol couse in adolescence on subsequent behaviour in adulthood are limited.

The adolescent brain undergoes critical neuronal and structural development, making adolescence a period of vulnerability to the effects of drugs $^{9}$, with brain imaging studies suggesting altered brain structure and function in adolescent users compared to nonusers $^{9}$. However, the causal consequences of adolescent co-use cannot be systematically examined in humans and thus, animal models are required to explore the 
cause-effect relationship. Although there is a dearth of pre-clinical studies that have examined the acute effects of adolescent nicotine and alcohol co-exposure, the research that has been conducted suggests their co-use to have additive effects on behavioural outcomes. In adolescent male rats, concurrent intravenous selfadministration of nicotine and alcohol was more reinforcing than either drug alone - an effect not observed in adults ${ }^{10}$. A combination of the two substances also increases ambulatory activity, and decreases anxiety-like behaviours in adolescent, but not adult, males ${ }^{11}$. Together, these results suggest that there may be fundamental differences in the effects of alcohol and nicotine co-use in adolescents compared to adults.

Despite the paucity of preclinical research that explores the long-term ramifications of adolescent nicotine and alcohol co-use, both drugs have been examined in isolation. Nicotine or alcohol exposure in adolescence increases the risk of substance use later in life, where alcohol exposure in adolescent rats increased voluntary ethanol drinking and preference in adulthood ${ }^{12}$, and adolescent nicotine-exposed rodents showed increased vulnerability to nicotine's reinforcing effects and enhanced reward responses to other drugs as adults ${ }^{13}$. These drug-induced neuroadaptations may result in the sensitization of reward-incentive processes such that reward-related stimuli acquire enhanced salience $^{14}$. Repeated exposure to alcohol or nicotine in adolescent rats increased conditioned approach toward reward-associated cues in adulthood ${ }^{15-17}$. Moreover, adult rodents exhibit long-term impairments across several cognitive domains as a consequence of alcohol exposure in adolescence, including spatial working memory ${ }^{18}$, object recognition memory ${ }^{19}$ and fear retention ${ }^{20}$. Similarly, rats treated with nicotine 
during adolescence display long-lasting dysfunctions in attention, impulsive behaviour ${ }^{21}$ and serial pattern learning ${ }^{22}$.

To date, no preclinical studies have directly tested the effects of adolescent alcohol drinking and nicotine vapour co-exposure on reward- and cognitive-related behaviours in adulthood. Given the known sex differences in the response to alcohol and nicotine ${ }^{23}$, as well as sex differences in brain development ${ }^{24}$, it is imperative to study these effects in both male and female rodents. We hypothesize that the co-exposure to nicotine vapour and alcohol in adolescence would produce pronounced changes in reward- and cognitive-associated behaviour when compared with exposure to either drug alone. Specifically, we predicted that the co-exposure to alcohol and vapourized nicotine would increase alcohol intake and preference in adulthood, enhance the incentive salience of reward-predictive stimuli and impair fear associative learning and memory.

\section{METHODS}

All procedures were approved by the Animal Care Committee at the University of Guelph under Canadian Council on Animal Care Guidelines. Male $(\mathrm{N}=39)$ and female $(\mathrm{N}=35)$ Sprague-Dawley rats aged post-natal day (PND) 21 upon arrival were obtained from Charles River (Montreal, Canada). The animals were maintained on a 12-hour light-dark cycle (7AM-7PM). Upon weaning (PND23), same-sex littermates were housed two per cage. On PND28, pair-housed rats were separated by a mesh divider in order to measure individual food, water and alcohol intake while still having social contact. All animals had access to standard chow and water ad libitum until behavioural testing 
commenced. The rats were randomly assigned to one of four exposure groups: 1) Vehicle vapour/Water (CO) (N=8/sex); 2) Vehicle/Alcohol (AO) (N=11/9, male/female); 3) Nicotine Vapour/Water (NO) ( $N=8 /$ sex) or 4) Nicotine Vapour/Alcohol (AN) ( $N=11 / 9$, male, female).

\section{Drug Preparation}

\section{Nicotine}

JUUL mint flavoured $5 \%$ nicotine e-liquid pods $(59 \mathrm{mg} / \mathrm{ml}$ ) (JUUL Labs, Toronto, Canada) or vehicle e-liquid without flavouring was administered. The vehicle e-liquid was a mixture of $30: 70$ propylene glycol to vegetable glycerine, representative of commercially available JUUL pods ${ }^{25}$.

Alcohol

Ethanol (Commercial Alcohols, Brampton, Canada) was diluted to a final concentration of $10 \% \mathrm{v} / \mathrm{v}$ in tap water ${ }^{12}$.

\section{Vapour Administration Procedure}

Beginning at PND30, passive nicotine vapour exposure was conducted using the OpenVape apparatus ${ }^{26}$. Animals in the nicotine vapour only (NO) and concurrent alcohol drinking and nicotine vapour (AN) exposure groups received nicotine vapour. Animals in the control (CO) and alcohol only (AO) exposure groups received vehicle vapour. All animals received 10 minutes of vapour exposure per day for 16 days, with each epoch of pump activation resulting in 10, 2-second puffs/minute ${ }^{26}$. Animals from each exposure group and sex were placed in the exposure chamber together. 


\section{Adolescent Alcohol Exposure}

During the duration of vapour exposure (PND30-46), rats assigned to $A O$ and $A N$ exposure groups were given 24-hour access to $10 \%$ ethanol and tap water in a twobottle preference design (described in detail below). Rats assigned to the $\mathrm{CO}$ and $\mathrm{NO}$ exposure groups received continuous access to two bottles of tap water.

\section{Two-Bottle Preference Test}

The effects of adolescent voluntary alcohol drinking (with or without nicotine vapour inhalation) on adulthood alcohol consumption and preference were quantified using a two-bottle alcohol preference test ${ }^{27}$. Beginning at PND110, all rats were given 24-hour access to $10 \%$ ethanol and tap water for four days. To prevent location preference development, the position of the bottles was switched daily. Bottle weights were recorded daily. The main outcome measurements were alcohol consumption (in $\mathrm{g} / \mathrm{kg}$ ) and alcohol preference. Alcohol preference was calculated as:

$\frac{\text { Intake of } 10 \% \text { Alcohol }}{\text { Intake of } 10 \% \text { Alcohol+Intake of } \mathrm{H} 2 \mathrm{O}} \times 100 \%$

\section{Effects of Adolescent Nicotine Vapour and Alcohol Co-Exposure an Reward- Related Behaviour an Adulthood}

In adulthood, body weights were reduced to $85 \%$ of baseline body weight. All rats remained food restricted until the completion of the experiment and were fed after each testing session. 


\section{Pavlovian Conditioned Approach Apparatus}

Pavlovian conditioned approach (PCA) task was used to measure the incentive salience of reward-predictive cues. Behavioural procedures were carried out in standard operant chambers (Coulbourn Instruments, Allentown, PA) enclosed in sound-attenuating cubicles $(76.2 \times 46.99 \times 44.96 \mathrm{~cm})$. The chambers were outfitted with a food cup located in the center of the right sidewall and retractable levers were positioned to the left and right of the food cup. The cubicles also contained surveillance cameras to monitor behaviours during testing. Data collection from Graphic State software (Coulbourn Instruments, Allentown, PA) was transformed using a customized Microsoft Excel macro.

\section{Behavioural Procedure}

All rats were given 45mg Dustless Precision sucrose banana flavoured pellets (BioServ, Flemington, NJ, product \#F0024) in their home cages for one day to reduce potential neophobia. On the first day of the experiment (PND85), each rat was assigned to a chamber and received one 30-minute magazine training session, during which a single banana pellet was freely delivered on a random time 30 -second schedule. PCA testing began 24-hours following magazine training and lasted 12 days. Each daily testing session was 60 minutes in duration and consisted of 25 conditioned stimulus (CS)+ and 25 CS- trials with an average inter-trial interval (ITI) of 60 seconds. CS+ trials consisted of a 10-second extension of a lever followed by the delivery of 2 banana pellets upon lever retraction. During CS- trials, a 10-second extension of the other lever 
occurred, but no reinforcer was delivered upon retraction. The presentation of the CS was pseudorandom such that no more than two of the same CS presentations could occur consecutively. The assignment of the left and right levers as either CS+ or CSwas counterbalanced across animals and within exposure groups.

\section{Effects of Adolescent Vapourized Nicotine and Alcohol Co-Exposure on Associative Memory in Adulthood}

Fear Conditioning Apparatus

Experiments were conducted in four standard fear-conditioning chambers (Med Associates Inc., St. Albans, VT; $29.53 \times 23.5 \times 20.96 \mathrm{~cm}$ ) enclosed in sound-attenuating cubicles $(63.5 \times 36.83 \times 74.93 \mathrm{~cm})$. A tone generator presented an auditory cue $(90 \mathrm{~dB}$, $2-\mathrm{kHz})$ as the CS. The steel bars were wired to a shock generator to deliver an electric foot shock $(1.0 \mathrm{~mA})$ as the unconditioned stimulus. Chambers were outfitted with either a lemon or vanilla scent and flat or zig-zag grid floors as contextual cues, with use of these cues counterbalanced across animals within each exposure group. Freezing behaviour, defined as total motor immobility except for movement necessitated by respiration ${ }^{28}$, was recorded and analyzed using Video Freeze Software. On the cue testing day, animals were introduced to a novel context arrangement (e.g., zig-zag grid floor substituted for flat grid floors, lemon substituted for vanilla scent) and a plastic sheet that rounded the chamber walls was inserted to provide a new context. 


\section{Behavioural Procedure}

As previously described ${ }^{29}$, the training session consisted of five 10-second tone presentations followed by foot-shock delivery during the last 2 seconds of the tone with an ITI of 64 seconds. Fear conditioning began on PND103 with the first trial starting 3 minutes after the rat is placed in the chamber. After a 24-hour period, freezing to the context was assessed by returning the rats to their original chamber for 8 minutes, with no tone or shock presented. One day later, fear conditioning to the tone was assessed by placing rats in a novel context. Following an initial 30-second delay, the tone was presented 20 times for 10-seconds each with a 30-second ITI between tone presentations. No shock was delivered to the animals. Incidences of freezing were recorded during the ITIs on training and tone test sessions, and in 64 second bins during the context test session ${ }^{29}$.

\section{Statistical Analyses}

Data analysis was performed using IBM SPSS Statistics 27 (Armonk, New York, United States) software. All data will be available upon publication at https://www.khokharlab.com/.

\section{Two-Bottle Preference Test Data Analysis}

Average alcohol intake and preference in adolescence and adulthood were compared both within- and between- ages using a 2-way between-subjects ANOVA with adolescent nicotine exposure and age as between-subjects factors, for males and females in groups exposed to alcohol in adolescence. Average alcohol intake and 
preference data from adulthood only was analyzed by a 2-way between-subject ANOVA with adolescent exposure and sex as between-subjects factors.

\section{PCA Behavioural Data Analysis}

Behavioural dependent measures included: (1) number of lever presses and food cup entries per session, (2) probability of pressing the lever and entering the food cup during a trial and (3) the latency to press the lever and enter the food cup during the 10-second CS presentation. Given previously observed sex differences in the impact of adolescent nicotine and alcohol exposure on behaviour ${ }^{22,23}$, males and females were analyzed separately for each behavioural measure using a 2-way-repeated measures factorial ANOVA with exposure (nicotine vapour and alcohol) as a between-subjects factor and day as a within-subject factor. Average PCA index scores (described below) for males and females were analyzed with a 2-way ANOVA with exposure (nicotine vapour and alcohol) as the between-subjects factor. Cohen's $d$ was also calculated as an estimation of effect size. The interpretation criteria for effect sizes were considered small, medium, or large if they corresponded to partial $\eta^{2}$ and Cohen's $d$ of at least $0.0099(d=0.29$ $0.49), 0.0588(d=0.50-0.79)$ and $0.13790(d=\geq 0.80)$, respectively.

\section{Quantification of Sign- and Goal-Tracking Behaviour}

A PCA index score was calculated to classify animals as goal-trackers, sign-trackers or intermediates based on average performance during all 12 sessions ${ }^{30}$. The PCA Index was calculated as the sum of (1) Response Bias (contacting the CS+ lever or food cup entries in relation to total number of $\mathrm{CS}_{+}$or food cup responses), (2) Probability 
Difference (difference between the probability of pressing the CS+ lever and probability entering the food cup) and (3) Latency Score (the difference between latency to contact the CS+ lever and latency to enter the food cup), which was then divided by 3 . Animals with scores of -1.0 to -0.3 were classified as goal-trackers and animals with scores of +0.3 to +1.0 were classified as sign-trackers. Animals that were within the range of 0.29 to +0.29 were defined as intermediates.

\section{Fear Conditioning Data Analysis}

Separate 2-way analyses were conducted for males and females given that they display distinct patterns of fear expression ${ }^{31}$. Freezing behaviour was analyzed using repeated measures ANOVA on each of the three days with adolescent exposure (nicotine vapour and alcohol) as the between-subjects factor and trial or block (64-second epoch) as the within-in subjects factor. One male animal in the $\mathrm{CO}$ and two male animals in the AN group were excluded due to their freezing being more than 2 standard deviations outside the mean.

\section{RESULTS}

\section{Two-Bottle Preference Test}

Female rats consumed more alcohol than males in adulthood, with both sexes showing greater alcohol preference in adulthood compared to adolescence.

No main effect of exposure or an exposure by sex interaction was revealed for alcohol intake or preference in adolescence or adulthood. A 2-way ANOVA comparing alcohol preference from adolescence and adulthood revealed a main effect of age for males 
$\left[F(1,40)=8.3 ; p=0.006, \eta_{p}^{2}=0.173\right]$ and females $\left[F(1,32)=30.8 ; p<0.05, \eta_{p}^{2}=\right.$ 0.491] where preference for alcohol was higher in adulthood relative to adolescence

(Figure 1 A,B). A significant main effect of age for average alcohol intake $(\mathrm{g} / \mathrm{kg})$ was observed in females $\left[F(1,32)=9.3 ; p=0.021, \eta_{p}^{2}=0.156\right]$ but not in males, indicating alcohol intake was higher in adulthood relative to intake in adolescence for female rats (Figure 1 C,D). An overall increase in alcohol intake $\left[F(1,66)=19.8 ; p<0.05, \eta_{p}^{2}=\right.$ $0.231]$ and preference $\left[F(1,66)=21.4 ; p<0.05, \eta_{p}^{2}=0.245\right]$ in adulthood was detected in female rats compared to male rats across all exposure groups (Figure $1 \mathbf{E , F}$ ).

\section{Pavlovian Conditioned Approach}

Male adolescent nicotine vapour exposure altered reward-associated learning in adulthood.

Sign-tracking was measured as lever pressing during CS+ presentation over the 12 testing sessions. Analysis of the number of lever presses revealed a main effect of session for males $\left[F(3.3,116.9)=11.4 ; p<0.05, \eta_{p}{ }^{2}=0.246\right]$ and females $[F(3.0,94.1)=$ 9.3; $p<0.05, \eta_{p}^{2}=0.231$ ] with lever pressing increasing across sessions. A main effect of nicotine vapour was reported for males $\left[F(1,35)=4.9 ; p=0.03, \eta_{p}^{2}=0.123\right]$ in the between-subject analysis, with both adolescent nicotine vapour-exposed male groups exhibiting significantly higher lever pressing compared to CO males $(d=1.0)$ (Figure 2A). No effect of nicotine vapour or alcohol drinking was observed in females (Figure 2B). Analysis of the probability to lever press revealed a main effect of session in males $\left[F(3.5,122.9)=25.6 ; p<0.05, n_{p}^{2}=0.422\right]$ and females $[F(4.0,124.1)=24.3 ; p<0.05$, $\left.\eta_{p}{ }^{2}=0.440\right]$ with the probability to press the lever increasing across sessions. A main 
effect of nicotine vapour exposure was revealed in males $\left[F(1,35)=9.5 ; p=0.004, \eta_{p}{ }^{2}=\right.$ 0.214] where both nicotine vapour exposed groups displayed a higher probability to press the lever compared to $\mathrm{CO}(d=1.2)$ and $\mathrm{AO}(d=0.8)$ exposure groups (Figure 2C). This effect was not observed in females (Figure 2D). Analysis of latency to lever press revealed a main effect of session in males $\left[F(3.4,117.8)=24.9 ; p<0.05, \eta_{p}{ }^{2}=\right.$ $0.416]$ and females $\left[F(4.0,123.4)=24.7 ; p<0.05, \eta_{p}{ }^{2}=0.443\right]$ with latency to press the lever decreasing across sessions. Male rats from both groups exposed to vapourized nicotine in adolescence demonstrated a shorter latency to press the lever $[F(1,35)=9.1$; $\left.\mathrm{p}=0.005, \eta_{\mathrm{p}}^{2}=0.206\right]$ relative to $\mathrm{CO}(d=1.2)$ and $\mathrm{AO}(d=0.8)$ exposure groups (Figure 2E). No differences were present amongst exposure groups in female rats (Figure 2F).

Goal-tracking was measured as food cup entries during CS+ presentation over the 12 testing sessions. Analysis of the number of food cup entries revealed a main effect of session for females $\left[F(3.5,108.9)=6.7 ; p<0.05, \eta_{p}{ }^{2}=0.177\right]$ but not males. There was no effect of nicotine vapour or alcohol exposure in either males or females (Figure 3 A,B). Analysis revealed a main effect of session for the probability and latency to enter the food cup in males $\left[F(2.4,85.6)=10.9 ; p<0.05, \eta_{p}{ }^{2}=0.237\right],[F(2.6,91.0)=24.2 ; p<$ $\left.0.05, \eta_{p}^{2}=0.408\right]$ and females $\left[F(2.4,74.9)=10.6 ; p<0.05, \eta_{p}^{2}=0.256\right],[F(3.1,96.2)=$ $\left.25 ; p<0.05, \eta_{p}{ }^{2}=0.446\right]$. An effect of nicotine vapour for males in the probability and latency to enter the food cup was observed $\left[F(1,35)=5.1 ; p=0.03, \eta_{p}^{2}=0.128\right]$, $\left[F(1,35)=6.6 ; p=0.02, \eta_{p}^{2}=0.158\right]$, with $A N$ exposed males demonstrating a lower probability and longer latency to enter the food cup compared to $\mathrm{CO}(d=1.1)$ and $\mathrm{AO}(d$ 
$=1.0$ ) exposed males (Figure $3 \mathbf{C}, \mathbf{E}$ ). In contrast, nicotine vapour exposure had no effect in females for either the probability or latency to enter the food cup (Figure 3 D,F). A repeated measures ANOVA for CS- approach revealed no significant effects of session and exposure across all behavioural metrics for males and females (data not shown).

Analysis of the average PCA index score revealed a main effect of nicotine vapour $\left[F(1,35)=8.4 ; p=0.006, \eta_{p}^{2}=0.194\right]$ in males exposed to vapourized nicotine. According to Cohen's criteria, a large effect was observed in the comparison between AN and CO $(d=1.2)$ exposed males, AN and AO $(d=0.9)$ exposed males, and between $\mathrm{NO}$ and $\mathrm{CO}(d=0.9)$ exposed males, where male groups exposed to vapourized nicotine had a higher PCA index score (Figure 4A). No such effect was observed in females (Figure 4B). Co-exposure of alcohol and nicotine increased the number of male sign-trackers to $63.6 \%$ relative to male controls of $0 \%$. Conversely, coexposure of alcohol and nicotine had no effect on the number of female sign-trackers relative to female controls with $22.2 \%$ of co-exposed females presenting with a signtracking phenotype compared to $25 \%$ of female controls (Table 1 ).

\section{Fear Conditioning}

Adolescent alcohol exposure in male rats impaired learning and contextual fear memory.

A main effect of post-shock trial for males $\left[F(3.6,116.4)=26.8 ; p<0.05, \eta_{p}^{2}=0.456\right]$ and females $\left[F(3.1,93.4)=22.5 ; p<0.05, \eta_{p}^{2}=0.428\right]$ was observed on the conditioning 
day, indicating a progressive increase in freezing behaviour as trials proceeded. During the conditioning session, a main effect of alcohol $\left[F(1,32)=9.2 ; p=0.005, \eta_{p}{ }^{2}=0.224\right]$, nicotine vapour $\left[F(1,32)=4.3 ; p=0.46, \eta_{p}{ }^{2}=0.119\right]$ and an alcohol-by-nicotine vapour interaction $\left[F(1,32)=8.3 ; p=0.007, \eta_{p}^{2}=0.206\right]$ was detected in males (Figure 5A), where male adolescent drug exposure resulted in significant deficits in fear acquisition relative to $\mathrm{CO}$ males. The level of post-shock freezing in females was comparable in all exposure groups during training (Figure 5B). Male and female rats in each group exhibited freezing during the context test, which diminished over the course of the session. This was confirmed by a repeated measures ANOVA in which there was a main effect of epoch in males $\left[F(4.3,138.9)=7.1 ; p<0.05, \eta_{p}^{2}=0.181\right]$ and females $\left[F(3.6,106.8)=5.7 ; p<0.05, \eta_{p}^{2}=0.160\right]$. Analysis revealed a main effect of alcohol in males $\left[F(1,32)=5.6 ; p=0.02, \eta_{p}^{2}=0.150\right]$ where $A O$ and $A N$ exposed males showed less freezing behaviour and demonstrated deficits in context-related memory (Figure 5C). There was no main effect of exposure on context-related memory for female rats (Figure 5D). A main effect of trial on cue testing day was revealed for males $\left[F(10.8,345.6)=8.3 ; p<0.05, \eta_{p}{ }^{2}=0.207\right]$ and females $\left[F(8.0,240.1)=7.2 ; p<0.05, \eta_{p}{ }^{2}\right.$ $=0.194]$, indicating both sexes demonstrated extinction of the fear response over repeated tone administration. No effect of exposure on cue-related memory was detected for either male or female rats (Figure 5 E,F).

\section{DISCUSSION}

Despite the epidemiological findings indicating frequent associations between ecigarette use and alcohol consumption ${ }^{4,5}$, there is a scarcity of information regarding the 
long-term behavioural consequences of their co-use during adolescence. Using a preclinical model of moderate voluntary adolescent alcohol intake and passive nicotine vapour administration, the current study found that exposure to alcohol and vapourized nicotine during this critical developmental period promotes unique sex-specific effects on incentive and associative learning processes.

The present study demonstrated adult male, but not female, rats exposed to vapourized nicotine in adolescence attributed greater incentive value to reward-predicting cues, evidenced by increased approach toward the cue (i.e., CS+), and were more likely to express a sign-tracking phenotype. The current findings confirm previous observations showing that subcutaneous nicotine injections during adolescence produce long-lasting alterations in reward-associated learning in adult male rats ${ }^{17}$. Animals that develop the conditioned response to approach the cue are termed sign-trackers, while others that preferentially approach the reward delivery location when the cue is presented are termed goal-trackers. Several studies have shown that exposure to substances of abuse foster sign-tracking behaviours ${ }^{15,16}$, with sign-tracking phenotypes being often associated with behaviours such as reduced impulse control and psychomotor sensitization ${ }^{32,33}$. Thus, exposure to vapourized nicotine during adolescence in males appears to produce a phenotype previously associated with addiction-vulnerability. Our study is also supported by clinical findings suggesting that nicotine use increases attentional bias to drug-associated cues in smokers ${ }^{34}$. The ability of nicotine vapour to enhance the incentive value of reward-predicting cues may be relevant to the initiation of future smoking. A meta-analysis revealed adolescent e-cigarette users had more 
than three times the odds of subsequent cigarette use and four times the odds of past 30-day smoking than non-users ${ }^{35}$. Additionally, in a human laboratory paradigm, the exposure to passive e-cigarette use increased adolescents urge to smoke a regular cigarette $^{36}$. Based on our observations and the aforementioned evidence, this suggests that e-cigarette use in adolescence poses as a major risk factor for future tobacco use and dependence.

Female rats appeared to be resistant to the long-lasting incentive-salience related effects observed in male rats. This contrasts previous findings that nicotine exposure in female rodents enhances the expression of sign-tracking behaviours ${ }^{37}$. However, these studies were performed in females injected with nicotine in adulthood, whereas repeated injection with nicotine in adolescence reduced approach behaviours in adult female rats $^{17}$. These sex differences highlight the potential impact of route of administration, as well as the age of exposure on the long-term effects of adolescent nicotine vapour exposure on reward-related behaviours. There is also evidence that hormones influence reward-associated behaviours. In females, the rewarding effects of substances of abuse are often elevated during times of low progesterone and high estrogen $^{38}$ and cues paired during the estrous stage have shown increased dopaminergic firing as opposed to the proestrus stage of the estrous cycle ${ }^{39}$. However, follow-up studies are needed to determine the mechanistic differences that underlie these sex-dependent behavioural effects. 
Conversely, adolescent exposure to alcohol had no effect on sign-tracking behaviours in adulthood. This adds to the mixed literature suggesting that prior alcohol exposure can lead to either an enhanced ${ }^{15,16,40}$ or absent ${ }^{16}$ sign-tracking responses. One factor contributing to this discrepancy might be differences in task design; previously, a divergence in behavioural responses between adolescent alcohol-exposed and control animals was detected by the $14^{\text {th }}$ day of testing ${ }^{40}$, requiring more training days than our study included. Interestingly, nicotine vapour and alcohol co-exposure led to similar behavioural profiles to nicotine vapour alone in the majority of the PCA measures, suggesting that a synergistic or additive effect of nicotine and alcohol was not present for these behaviours and their combined effect was primarily driven by the actions of nicotine vapour. However, with respect to goal-tracking, males co-exposed to nicotine vapour and alcohol during adolescence, but not nicotine vapour alone, showed decreased goal-tracking behaviours relative to males exposed to alcohol alone, suggesting a potentiation of nicotine vapour's effects by alcohol. Consistently, coadministration of alcohol and nicotine produces an additive release of dopamine in the nucleus accumbens core compared to each drug in isolation ${ }^{41}$, as well as behavioural disruptions not observed following alcohol administration exclusively ${ }^{42}$.

Male, but not female, rats exposed to alcohol, nicotine or the combination during adolescence exhibited a deficit in fear acquisition compared to controls as denoted by a reduction in the percentage of freezing time across the post-shock trials. These results are consistent with previous work suggesting that sex moderates the effects of adolescent drug exposure on cognitive function ${ }^{43}$. The reduced fear response in 
adolescent drug-exposed males may hinge on impairments in underlying learning mechanisms. Specifically, the amygdala plays a role in the acquisition of fear memory ${ }^{44}$, and drug use in adolescence has been documented to disrupt amygdala processes that persist into adulthood ${ }^{45}$, suggesting that the amygdala may be particularly vulnerable to adolescent drug exposure. The amygdala is also implicated in tone fear conditioning; however, we found adolescent drug exposure did not influence behaviour to the tone presented alone. The several nuclei that make up the amygdala each mediate different types of conditioned fear behaviour ${ }^{46}$. Lesions to the basolateral nuclei produce deficits in conditioned fear acquisition, while not altering auditory-cue conditioning ${ }^{47}$. This implies that the impairments we observed in fear acquisition, but not tone-induced freezing, might be a result of drug-induced insult to only the basolateral nuclei while sparing other nuclei necessary for tone cue-conditioning. Our results are in agreement with prior studies that found no differences in tone freezing responses in adult rats exposed to alcohol or nicotine during adolescence ${ }^{20,48}$. Adolescent alcohol-exposed male rats showed contextual memory deficits in adulthood, which is dependent on the hippocampus $^{49}$. However, given that alcohol exposure compromised fear acquisition, the context memory deficits may not be due to memory retention, but a result of learning impairments. Nonetheless, these effects corroborate previous literature that have reported alcohol-induced disruptions of contextual fear conditioning during adolescence ${ }^{20}$.

In contrast to previous findings with nicotine ${ }^{48,50}$, we found no effect of adolescent nicotine vapour exposure during contextual fear conditioning in either male or female 
rats. However, these inconsistencies may be a result of differing study parameters such as the strain of animal, dosage used, route of administration, and dependent measure to assess fear conditioning. In fact, studies utilizing freezing as the dependent measure produced a similar lack of effects ${ }^{51}$, whereas lick suppression protocols showed adolescent nicotine-induced deficits ${ }^{50}$. These procedural differences could therefore hinder study comparisons and limit the development of hypotheses related to the effects of vaporized nicotine. As noted earlier, male rats expressed enhanced freezing behaviours compared to female rats. However, these discrepancies may not reflect genuine learning and memory deficits in females, but rather be a product of our fear assessment method. Male rats are more likely to perform inactive responses such as freezing, while females engage in active responses such as darting representing escape-like behaviour, therefore exhibiting lower levels of freezing ${ }^{31}$. For that reason, future studies should utilize multiple indices of fear behaviours, particularly when comparing sexes. While the exact neurobiological basis that underlies this sexual dimorphism remains unknown, these findings add to our current understanding regarding the role of sex and drug use in learning and memory.

Contrary to our predictions, we found that the exposure to nicotine vapour, alcohol or its combination in adolescence had no effect on alcohol intake or preference in adulthood. These results are in agreement with previous findings on adolescent nicotine and alcohol exposure that enforced voluntary access to alcohol in adulthood ${ }^{52,53}$. However, there are inconsistencies throughout the literature, where previous studies have shown early exposure to alcohol either increased ${ }^{12,54}$ or decreased ${ }^{27}$ subsequent alcohol $^{2}$ 
intake. Additionally, both the exposure to nicotine and the co-exposure of nicotine and alcohol in adolescence has been reported to enhance alcohol drinking later in life ${ }^{10}$. These conflicting patterns of responses may be dependent on methodological distinctions such as regimen of administration during adolescence and choice of alcohol consumption paradigms used in adulthood. For instance, forced alcohol exposure has been shown to induce stress as opposed to voluntary access, and as a result increases alcohol preference ${ }^{53}$. Moreover, taste aversion to alcohol may account for the lack of increased alcohol consumption in the present study. Indeed, it is well established that alcohol induces an aversive effect in rodents causing the restriction of ingestion, even in those selectively bred to prefer alcohol..$^{55}$

Consistent with previous research ${ }^{54}$, we found that female rats consumed significantly more alcohol than male rats. A potential explanation for this sex-specific pattern may be the fact that female rats are less sensitive to the hypnotic effects of alcohol relative to males ${ }^{56}$. This insensitivity would serve as a permissive feature, leading females to consume more alcohol before experiencing pharmacological feedback that would moderate their intake. Prior studies have suggested potential impacts of the female reproductive cycle and fluctuating hormones on alcohol intake with changes in the reinforcing properties of alcohol throughout the estrous cycle in rats with synchronized cycles $^{56}$ while others have reported no impact in freely cycling rats ${ }^{57}$. Though we cannot be certain that our female rats are asynchronous, we saw no day-to-day variations in their drinking behaviours that would indicate a change in alcohols reinforcing effects. Thus, the present results suggest that gonadal hormone fluctuations may have minimal 
influence on the consumption of alcohol in our female rodents. Although animals exposed to alcohol in adolescence did not differ from controls in alcohol consumption during adulthood, within-animal differences in the consumption of alcohol during adolescence compared to later drinking demonstrated increases in preference for both male and female rats. It was also observed that female rats consumed more alcohol in adulthood compared to their intake in adolescence. Given the prominent brain development that occurs during adolescence, it is likely that alcohol sensitizes neuronal circuits involved in reinforcement (i.e. mesocorticolimbic pathway) that increases the risk of alcohol-related issues later in life ${ }^{58}$. Additionally, these results may suggest that adult consumption is related to solution familiarity as opposed to the effects of adolescent alcohol exposure itself. When interpreted this way, our data is in accordance with previous findings that have reported enhancement of adult alcohol consumption relative to drinking during adolescence due to solution acceptance ${ }^{59}$. Taken together with the literature, our findings indicate that the effect of adolescent exposure to nicotine vapour, alcohol or the combination in regulating alcohol consumption in adulthood heavily depends on multiple variables, and the complexity of these findings highlight the significance in considering intra-individual differences as opposed to solely group differences in such analyses.

The present study is the first to investigate the long-term sex-specific effects of adolescent concurrent nicotine vapour and alcohol exposure on subsequent behaviours, showing that adolescent alcohol drinking, vapourized nicotine and their combined exposure impact reward-driven and cognitive-associated behaviours later in life. We did 
not find additive effects of the combination of alcohol drinking and nicotine vapour; it is likely that alternate dose combinations may be needed to observe a drug interaction. Furthermore, we did not assess the impact of co-exposure on the pharmacokinetics and pharmacodynamics of individual drugs, and how they may be influenced by age or sex. This is of critical importance given the majority of our findings were sex-specific. Future studies incorporating assessments of plasma drug levels and receptor expression should be conducted to provide better insight into these observed differences. Nevertheless, our results add to the growing list of findings that highlight the sex-related variations that emerge and call attention to the importance of utilizing both sexes when measuring behavioural and cognitive outcomes. With the recent escalation of ecigarette use among teens and its association with the consumption of alcohol, these findings underscore the importance of studying the causal consequences of e-cigarette and alcohol co-use during adolescence, and ultimately elucidate the neurobiological underpinnings that drive the effects of these drugs.

\section{Acknowledgments}

JR, JAF, HHAT and JYK conceptualized the paper. JR, JAF, HHAT and MAT performed experiments. JR and JAF performed analyses. JR wrote the manuscript and JYK, JAF and HHAT provided manuscript revisions and finalized the manuscript for submission; all authors have given feedback on the final manuscript and approved its submission.

\section{Declaration of Interest}

No potential conflicts of interest were disclosed. 


\section{Funding}

This work was supported by the Canadian Institutes of Health Research (CIHR) Catalyst (Grant Number 442011) awarded to JYK and supported by CIHR Vanier Graduate Scholarship awarded to JAF. 


\section{References}

1. Miech R, Leventhal A, Johnston L, O'Malley PM, Patrick ME, Barrington-Trimis J. Trends in Use and Perceptions of Nicotine Vaping Among US Youth From 2017 to 2020. JAMA Pediatr 2021;175(2):185-190. (In eng). DOI: 10.1001/jamapediatrics.2020.5667.

2. Swendsen J, Burstein M, Case B, et al. Use and abuse of alcohol and illicit drugs in US adolescents: results of the National Comorbidity Survey-Adolescent Supplement. Arch Gen Psychiatry 2012;69(4):390-8. (In eng). DOI: 10.1001/archgenpsychiatry.2011.1503.

3. Crummy EA, O'Neal TJ, Baskin BM, Ferguson SM. One Is Not Enough: Understanding and Modeling Polysubstance Use. Front Neurosci 2020;14:569. (In eng). DOI: 10.3389/fnins.2020.00569.

4. Zuckermann AME, Williams G, Battista K, de Groh M, Jiang Y, Leatherdale ST. Trends of poly-substance use among Canadian youth. Addict Behav Rep 2019;10:100189. (In eng). DOI: 10.1016/j.abrep.2019.100189.

5. Rothrock AN, Andris H, Swetland SB, et al. Association of E-cigarettes with adolescent alcohol use and binge drinking-drunkenness: A systematic review and meta-analysis. Am J Drug Alcohol Abuse 2020;46(6):684-698. (In eng). DOI: 10.1080/00952990.2020.1771723.

6. Frie JA NC, Murray JE, Khokhar JY. Addiction-Related Outcomes of Nicotine and Alcohol Co-use: New Insights Following the Rise in Vaping. Nicotine \& Tobacco Research 2021.

7. Marshall AM, Heffernan T, Hamilton C. The Synergistic Impact of Excessive Alcohol Drinking and Cigarette Smoking upon Prospective Memory. Front Psychiatry 2016;7:75. (In eng). DOI: 10.3389/fpsyt.2016.00075.

8. Durazzo TC, Mon A, Gazdzinski S, Meyerhoff DJ. Chronic cigarette smoking in alcohol dependence: associations with cortical thickness and $\mathrm{N}$-acetylaspartate levels in the extended brain reward system. Addict Biol 2013;18(2):379-91. (In eng). DOI: 10.1111/j.1369-1600.2011.00407.x.

9. Hamidullah S, Thorpe HHA, Frie JA, McCurdy RD, Khokhar JY. Adolescent Substance Use and the Brain: Behavioral, Cognitive and Neuroimaging Correlates. Front Hum Neurosci 2020;14:298. (In eng). DOI: 10.3389/fnhum.2020.00298.

10. Lárraga A, Belluzzi JD, Leslie FM. Nicotine Increases Alcohol Intake in Adolescent Male Rats. Front Behav Neurosci 2017;11:25. (In eng). DOI: 10.3389/fnbeh.2017.00025.

11. Cross SJ, Leslie FM. Combined nicotine and ethanol age-dependently alter neural and behavioral responses in male rats. Behav Pharmacol 2021 (In eng). DOI: 10.1097/fbp.0000000000000622.

12. Alaux-Cantin S, Warnault $\mathrm{V}$, Legastelois $\mathrm{R}$, et al. Alcohol intoxications during adolescence increase motivation for alcohol in adult rats and induce neuroadaptations in the nucleus accumbens. Neuropharmacology 2013;67:52131. DOI: 10.1016/j.neuropharm.2012.12.007. 
13. Alajaji M, Lazenka MF, Kota $D$, et al. Early adolescent nicotine exposure affects later-life cocaine reward in mice. Neuropharmacology 2016;105:308-17. DOI: 10.1016/j.neuropharm.2016.01.032.

14. Berridge KC, Robinson TE. Liking, wanting, and the incentive-sensitization theory of addiction. Am Psychol 2016;71(8):670-679. (In eng). DOI: 10.1037/amp0000059.

15. McClory AJ, Spear LP. Effects of ethanol exposure during adolescence or in adulthood on Pavlovian conditioned approach in Sprague-Dawley rats. Alcohol 2014;48(8):755-63. (In eng). DOI: 10.1016/j.alcohol.2014.05.006.

16. Madayag AC, Stringfield SJ, Reissner KJ, Boettiger CA, Robinson DL. Sex and Adolescent Ethanol Exposure Influence Pavlovian Conditioned Approach. Alcohol Clin Exp Res 2017;41(4):846-856. (In eng). DOI: 10.1111/acer.13354.

17. Quick SL, Olausson P, Addy NA, Taylor JR. Repeated nicotine exposure during adolescence alters reward-related learning in male and female rats. Behav Brain Res 2014;261:171-6. (In eng). DOI: 10.1016/j.bbr.2013.12.001.

18. White AM, Ghia AJ, Levin ED, Swartzwelder HS. Binge pattern ethanol exposure in adolescent and adult rats: differential impact on subsequent responsiveness to ethanol. Alcohol Clin Exp Res 2000;24(8):1251-6. (In eng).

19. Fernandes LMP, Cartágenes SC, Barros MA, et al. Repeated cycles of binge-like ethanol exposure induce immediate and delayed neurobehavioral changes and hippocampal dysfunction in adolescent female rats. Behav Brain Res 2018;350:99-108. (In eng). DOI: 10.1016/j.bbr.2018.05.007.

20. Broadwater M, Spear LP. Consequences of adolescent or adult ethanol exposure on tone and context fear retention: effects of an acute ethanol challenge during conditioning. Alcohol Clin Exp Res 2014;38(5):1454-60. (In eng). DOI: 10.1111/acer.12382.

21. Counotte DS, Spijker S, Van de Burgwal LH, et al. Long-lasting cognitive deficits resulting from adolescent nicotine exposure in rats. Neuropsychopharmacology 2009;34(2):299-306. (In eng). DOI: 10.1038/npp.2008.96.

22. Fountain SB, Rowan JD, Kelley BM, Willey AR, Nolley EP. Adolescent exposure to nicotine impairs adult serial pattern learning in rats. Exp Brain Res 2008;187(4):651-6. (In eng). DOI: 10.1007/s00221-008-1346-4.

23. Kasten CR, Carzoli KL, Sharfman NM, et al. Adolescent alcohol exposure produces sex differences in negative affect-like behavior and group I mGluR BNST plasticity. Neuropsychopharmacology 2020;45(8):1306-1315. (In eng). DOI: 10.1038/s41386-020-0670-7.

24. Kaczkurkin AN, Raznahan A, Satterthwaite TD. Sex differences in the developing brain: insights from multimodal neuroimaging. Neuropsychopharmacology 2019;44(1):71-85. (In eng). DOI: 10.1038/s41386-018-0111-z.

25. Talih S, Salman R, El-Hage R, et al. Characteristics and toxicant emissions of JUUL electronic cigarettes. Tob Control 2019;28(6):678-680. (In eng). DOI: 10.1136/tobaccocontrol-2018-054616.

26. Frie JA, Underhill J, Zhao B, de Guglielmo G, Tyndale RF, Khokhar JY. OpenVape: An Open-Source E-Cigarette Vapor Exposure Device for Rodents. eNeuro 2020;7(5) (In eng). DOI: 10.1523/eneuro.0279-20.2020. 
27. Hamidullah S, Lutelmowski CD, Creighton SD, et al. Effects of vapourized THC and voluntary alcohol drinking during adolescence on cognition, reward, and anxiety-like behaviours in rats. Prog Neuropsychopharmacol Biol Psychiatry 2021;106:110141. (In eng). DOI: 10.1016/j.pnpbp.2020.110141.

28. Fanselow MS. Conditioned and unconditional components of post-shock freezing. Pavlov J Biol Sci 1980;15(4):177-82. (In eng). DOI: $10.1007 /$ bf03001163.

29. Keene CS, Bucci DJ. Neurotoxic lesions of retrosplenial cortex disrupt signaled and unsignaled contextual fear conditioning. Behav Neurosci 2008;122(5):10707. (In eng). DOI: 10.1037/a0012895.

30. Meyer PJ, Lovic V, Saunders BT, et al. Quantifying Individual Variation in the Propensity to Attribute Incentive Salience to Reward Cues. PLOS ONE 2012;7(6):e38987. DOI: 10.1371/journal.pone.0038987.

31. Gruene TM, Flick K, Stefano A, Shea SD, Shansky RM. Sexually divergent expression of active and passive conditioned fear responses in rats. Elife 2015;4 (In eng). DOI: 10.7554/eLife.11352.

32. Flagel SB, Watson SJ, Robinson TE, Akil H. Individual differences in the propensity to approach signals vs goals promote different adaptations in the dopamine system of rats. Psychopharmacology (Berl) 2007;191(3):599-607. (In eng). DOI: 10.1007/s00213-006-0535-8.

33. Tomie A, Grimes KL, Pohorecky LA. Behavioral characteristics and neurobiological substrates shared by Pavlovian sign-tracking and drug abuse. Brain Res Rev 2008;58(1):121-35. (In eng). DOI: 10.1016/j.brainresrev.2007.12.003.

34. Chanon VW, Sours CR, Boettiger CA. Attentional bias toward cigarette cues in active smokers. Psychopharmacology (Berl) 2010;212(3):309-20. (In eng). DOI: 10.1007/s00213-010-1953-1.

35. Soneji S, Barrington-Trimis JL, Wills TA, et al. Association Between Initial Use of e-Cigarettes and Subsequent Cigarette Smoking Among Adolescents and Young Adults: A Systematic Review and Meta-analysis. JAMA Pediatr 2017;171(8):788797. (In eng). DOI: 10.1001/jamapediatrics.2017.1488.

36. King AC, Smith LJ, McNamara PJ, Matthews AK, Fridberg DJ. Passive exposure to electronic cigarette (e-cigarette) use increases desire for combustible and ecigarettes in young adult smokers. Tob Control 2015;24(5):501-4. (In eng). DOI: 10.1136/tobaccocontrol-2014-051563.

37. Stringfield SJ, Madayag AC, Boettiger CA, Robinson DL. Sex differences in nicotine-enhanced Pavlovian conditioned approach in rats. Biol Sex Differ 2019;10(1):37. (In eng). DOI: 10.1186/s13293-019-0244-8.

38. Lynch WJ, Sofuoglu M. Role of progesterone in nicotine addiction: evidence from initiation to relapse. Exp Clin Psychopharmacol 2010;18(6):451-61. (In eng). DOI: 10.1037/a0021265.

39. Zhang D, Yang S, Yang C, Jin G, Zhen X. Estrogen regulates responses of dopamine neurons in the ventral tegmental area to cocaine. Psychopharmacology (Berl) 2008;199(4):625-35. (In eng). DOI: 10.1007/s00213008-1188-6. 
40. Spoelder M, Tsutsui KT, Lesscher HM, Vanderschuren LJ, Clark JJ. Adolescent Alcohol Exposure Amplifies the Incentive Value of Reward-Predictive Cues Through Potentiation of Phasic Dopamine Signaling. Neuropsychopharmacology 2015;40(13):2873-85. (In eng). DOI: 10.1038/npp.2015.139.

41. Tizabi Y, Bai L, Copeland RL, Jr., Taylor RE. Combined effects of systemic alcohol and nicotine on dopamine release in the nucleus accumbens shell. Alcohol Alcohol 2007;42(5):413-6. (In eng). DOI: 10.1093/alcalc/agm057.

42. Rezvani AH, Levin ED. Nicotine-alcohol interactions and cognitive function in rats. Pharmacol Biochem Behav 2002;72(4):865-72. (In eng). DOI: 10.1016/s0091-3057(02)00762-1.

43. Noorbakhsh S, Afzali MH, Boers E, Conrod PJ. Cognitive Function Impairments Linked to Alcohol and Cannabis Use During Adolescence: A Study of Gender Differences. Front Hum Neurosci 2020;14:95. (In eng). DOI: 10.3389/fnhum.2020.00095.

44. Fendt M, Fanselow MS. The neuroanatomical and neurochemical basis of conditioned fear. Neurosci Biobehav Rev 1999;23(5):743-60. (In eng). DOI: 10.1016/s0149-7634(99)00016-0.

45. Kyzar EJ, Zhang H, Sakharkar AJ, Pandey SC. Adolescent alcohol exposure alters lysine demethylase 1 (LSD1) expression and histone methylation in the amygdala during adulthood. Addict Biol 2017;22(5):1191-1204. (In eng). DOI: 10.1111/adb.12404.

46. Killcross S, Robbins TW, Everitt BJ. Different types of fear-conditioned behaviour mediated by separate nuclei within amygdala. Nature 1997;388(6640):377-80. (In eng). DOI: $10.1038 / 41097$.

47. Goosens KA, Maren S. Contextual and auditory fear conditioning are mediated by the lateral, basal, and central amygdaloid nuclei in rats. Learn Mem 2001;8(3):148-55. (In eng). DOI: 10.1101/lm.37601.

48. Portugal GS, Wilkinson DS, Turner JR, Blendy JA, Gould TJ. Developmental effects of acute, chronic, and withdrawal from chronic nicotine on fear conditioning. Neurobiol Learn Mem 2012;97(4):482-94. (In eng). DOI: 10.1016/j.nlm.2012.04.003.

49. Sanders MJ, Wiltgen BJ, Fanselow MS. The place of the hippocampus in fear conditioning. Eur J Pharmacol 2003;463(1-3):217-23. (In eng). DOI: 10.1016/s0014-2999(03)01283-4.

50. Spaeth AM, Barnet RC, Hunt PS, Burk JA. Adolescent nicotine exposure disrupts context conditioning in adulthood in rats. Pharmacol Biochem Behav 2010;96(4):501-6. (In eng). DOI: 10.1016/j.pbb.2010.07.011.

51. Smith LN, McDonald CG, Bergstrom HC, et al. Long-term changes in fear conditioning and anxiety-like behavior following nicotine exposure in adult versus adolescent rats. Pharmacol Biochem Behav 2006;85(1):91-7. (In eng). DOI: 10.1016/j.pbb.2006.07.014.

52. Smith AM, Kelly RB, Chen WJ. Chronic continuous nicotine exposure during periadolescence does not increase ethanol intake during adulthood in rats. Alcohol Clin Exp Res 2002;26(7):976-9. (In eng). DOI: 10.1097/01.Alc.0000021176.13538.55. 
53. Siegmund S, Vengeliene V, Singer MV, Spanagel R. Influence of age at drinking onset on long-term ethanol self-administration with deprivation and stress phases. Alcohol Clin Exp Res 2005;29(7):1139-45. (In eng). DOI: 10.1097/01.alc.0000171928.40418.46.

54. Amodeo LR, Kneiber D, Wills DN, Ehlers CL. Alcohol drinking during adolescence increases consumptive responses to alcohol in adulthood in Wistar rats. Alcohol 2017;59:43-51. (In eng). DOI: 10.1016/j.alcohol.2016.12.002.

55. McKinzie DL, Eha R, Murphy JM, McBride WJ, Lumeng L, Li TK. Effects of taste aversion training on the acquisition of alcohol drinking in adolescent $P$ and $H A D$ rat lines. Alcohol Clin Exp Res 1996;20(4):682-7. (In eng). DOI: 10.1111/j.15300277.1996.tb01672.x.

56. Cha YM, Li Q, Wilson WA, Swartzwelder HS. Sedative and GABAergic effects of ethanol on male and female rats. Alcohol Clin Exp Res 2006;30(1):113-8. (In eng). DOI: 10.1111/j.1530-0277.2006.00005.x.

57. Maldonado-Devincci AM, Badanich KA, Kirstein CL. Alcohol during adolescence selectively alters immediate and long-term behavior and neurochemistry. Alcohol 2010;44(1):57-66. (In eng). DOI: 10.1016/j.alcohol.2009.09.035.

58. Pascual M, Boix J, Felipo V, Guerri C. Repeated alcohol administration during adolescence causes changes in the mesolimbic dopaminergic and glutamatergic systems and promotes alcohol intake in the adult rat. $\mathrm{J}$ Neurochem 2009;108(4):920-31. (In eng). DOI: 10.1111/j.1471-4159.2008.05835.x.

59. Broadwater M, Varlinskaya EI, Spear LP. Effects of voluntary access to sweetened ethanol during adolescence on intake in adulthood. Alcohol Clin Exp Res 2013;37(6):1048-55. (In eng). DOI: 10.1111/acer.12049. 
Table 1. PCA Index Scores

\begin{tabular}{|c|c|c|c|c|c|c|c|c|c|}
\hline \multirow[t]{2}{*}{ Male } & \multicolumn{9}{|c|}{ Female } \\
\hline & Co & AO & NO & AN & & co & AO & NO & AN \\
\hline GT & 50 & 45.4 & 22.2 & 27.3 & GT & 50 & 22.2 & 33.3 & 22.2 \\
\hline Int. & 50 & 36.4 & 33.3 & 9.1 & Int. & 25 & 22.2 & 22.2 & 55.6 \\
\hline ST & 0 & 18.2 & 44.5 & 63.6 & ST & 25 & 55.6 & 44.5 & 22.2 \\
\hline
\end{tabular}

Data are presented as percentage of animals in each exposure group exhibiting goal tracking (GT), intermediate (Int.) or sign tracking (ST) phenotypes.

\section{Figure Legends}

Figure 1. Effects of adolescent nicotine vapour and alcohol exposure on alcohol drinking in adult male and female rats. On average, males A) and females B) in AO and AN exposure groups had a higher alcohol preference during adulthood relative to their preference in adolescence. C) There were no differences in alcohol consumption in males across age. D) Females consumed more alcohol as adults compared to their consumption as adolescents. On average, females E) drank significantly more alcohol in adulthood and F) had a higher preference for alcohol compared to males across all exposure groups. The data is presented as mean \pm SEM. ${ }^{*} p \leq 0.05$ significant differences between A, B, D) adolescent and adult drinking behaviours and E, F) male and female drinking behaviours.

Figure 2. Effects of adolescent nicotine vapour and alcohol exposure on sign-tracking in adult male and female rats. Males exposed to nicotine vapour as adolescents showed A) increased lever pressing compared to $\mathrm{CO}$ males $\mathrm{C}$ ) increased probability to press the lever compared to $\mathrm{CO}$ and $\mathrm{AO}$ males and E) a shorter latency to press the lever compared to $\mathrm{CO}$ and $\mathrm{AO}$ males. B, D, F) No differences were observed amongst female exposure groups in sign-tracking measures. The data is presented as mean \pm SEM. * $p$ $\leq 0.05$ significant difference between males that received vapourized nicotine and males that did not receive vapourized nicotine in adolescence.

Figure 3. Effects of adolescent nicotine vapour and alcohol exposure on goal-tracking in adult male and female rats. A) No exposure group differences in the number of food cup entries was observed amongst males. Males exposed to nicotine vapour as adolescents showed C) decreased probability and E) an increased latency to enter the food cup, with a strong effect detected between AN males vs. $\mathrm{CO}$ and $\mathrm{AO}$ males. B, D, F) No differences were observed amongst female exposure groups in goal-tracking measures. The data is presented as mean \pm SEM. ${ }^{*} p \leq 0.05$ significant difference 
between males that received vapourized nicotine and males that did not receive vapourized nicotine in adolescence.

Figure 4. Lever press and food cup entry number, probability and latency were combined into a PCA index score for each session and averaged over the 12 PCA sessions. PCA index scores are used to classify rats as sign-trackers (STs) (score +0.3 to +1.0), intermediates (Int.) (score -0.29 to +0.29 ) and goal-trackers (GTs) (score -1.0 to -0.3). A) male groups exposed to vapourized nicotine in adolescence had a higher PCA index score compared to $\mathrm{CO}$ males. B) No significant differences for PCA index scores were detected in females. The data is presented as mean \pm SEM. ${ }^{*} p \leq 0.05$ significant difference between males that received vapourized nicotine and males that did not receive vapourized nicotine in adolescence.

Figure 5. Effects of adolescent drug exposure on freezing behaviour in male and female rats. Adolescent drug exposure significantly impaired fear acquisition in A) males but not in B) females. C) Male groups exposed to alcohol showed impairments in contextual fear memory. D) No significant effects were detected in females. E, F) Adolescent drug exposure had no significant effects on freezing in males or females during the tone test session. Data is presented as mean \pm SEM. * $p \leq 0.05$ significant difference between $\mathrm{A}$ ) male exposure groups and $\mathrm{CO}$ males and $\mathrm{C}$ ) $\mathrm{AO}$ and $\mathrm{AN}$ males compared to $\mathrm{CO}$ males. 
bioRxiv preprint doi: https://doi.org/10.1101/2021.06.23.449629; this version posted June 24, 2021. The copyright holder for this preprint (which was not certified by peer review) is the author/funder. All rights reserved. No reuse allowed without permission. 

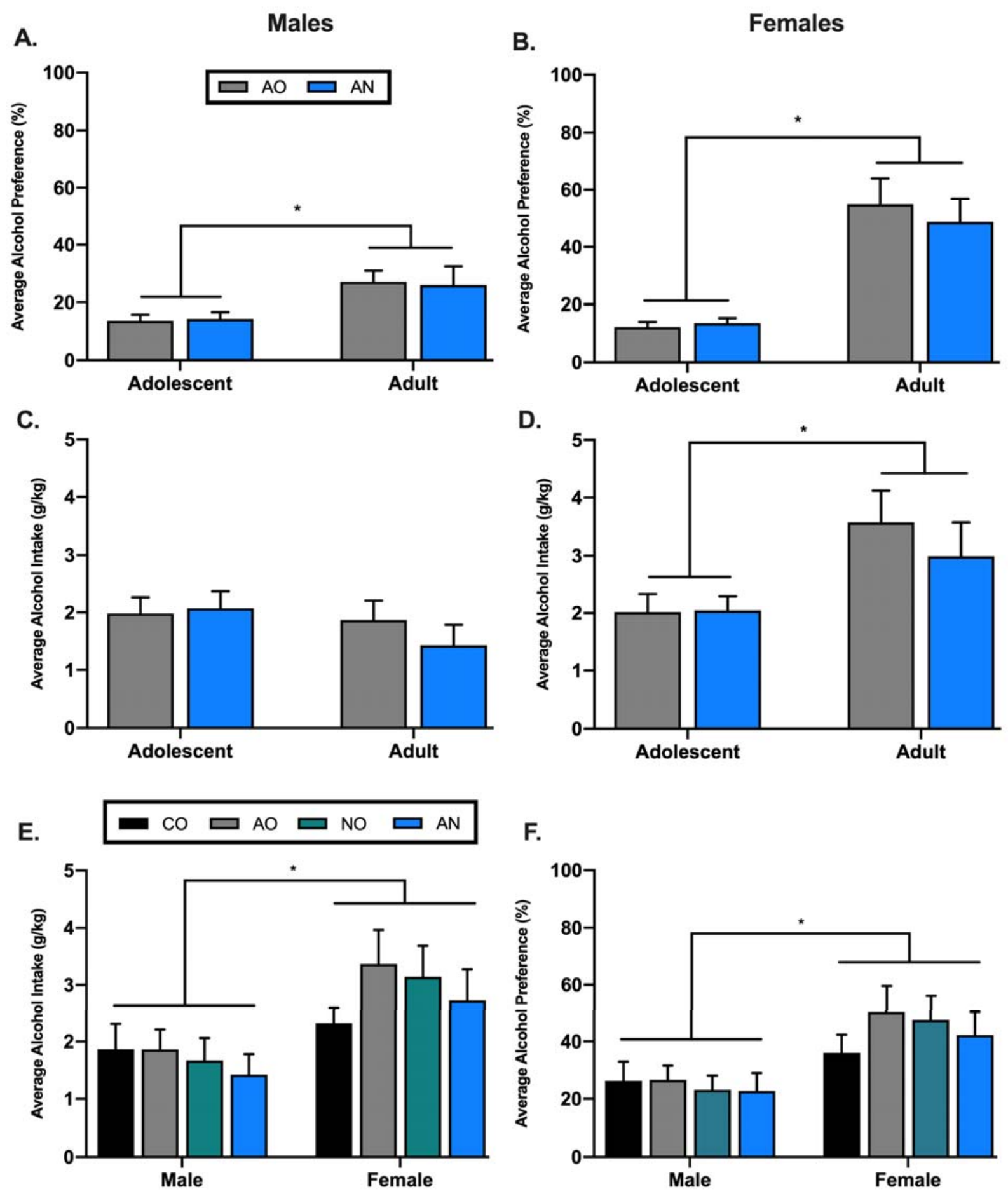

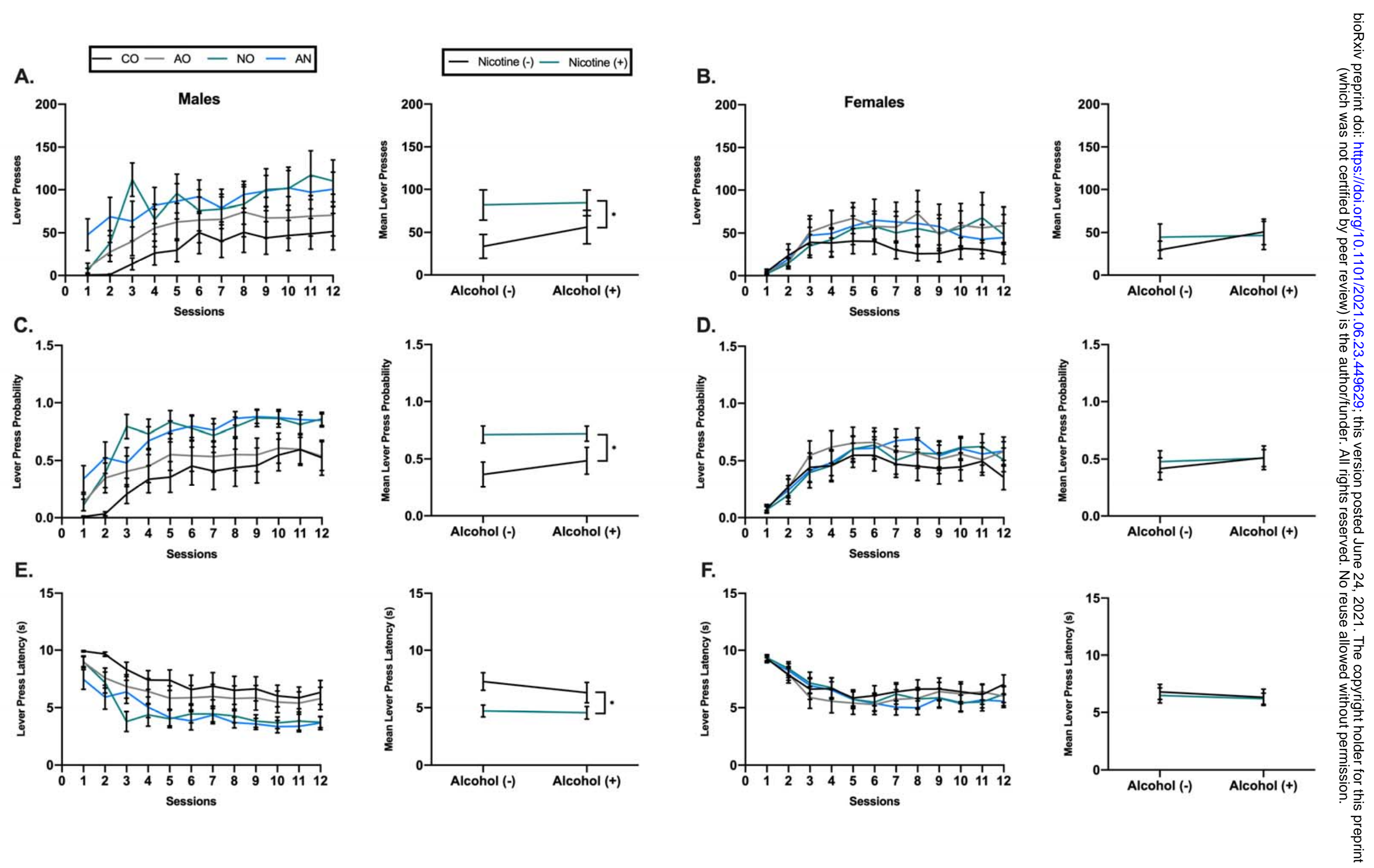

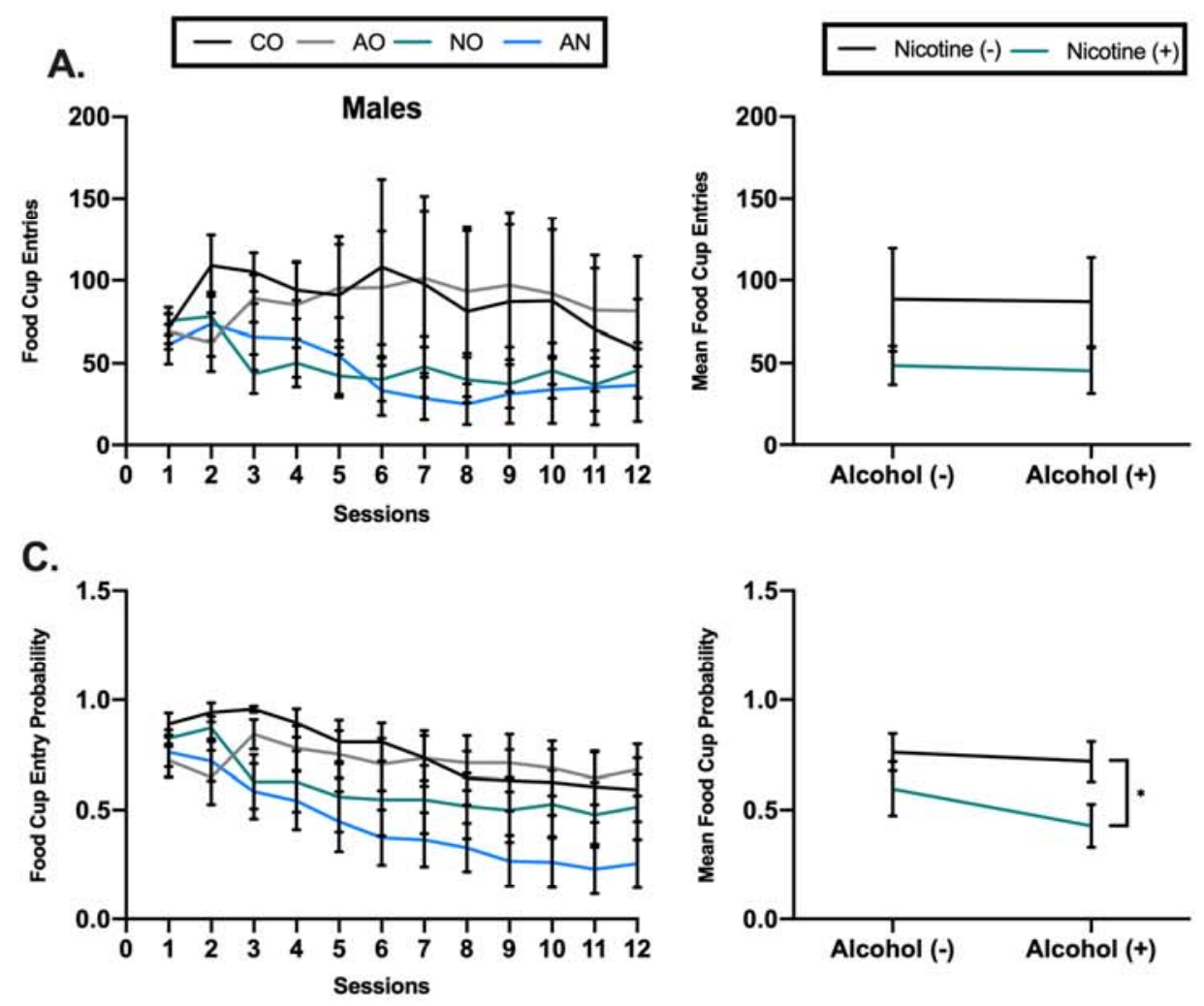

B.
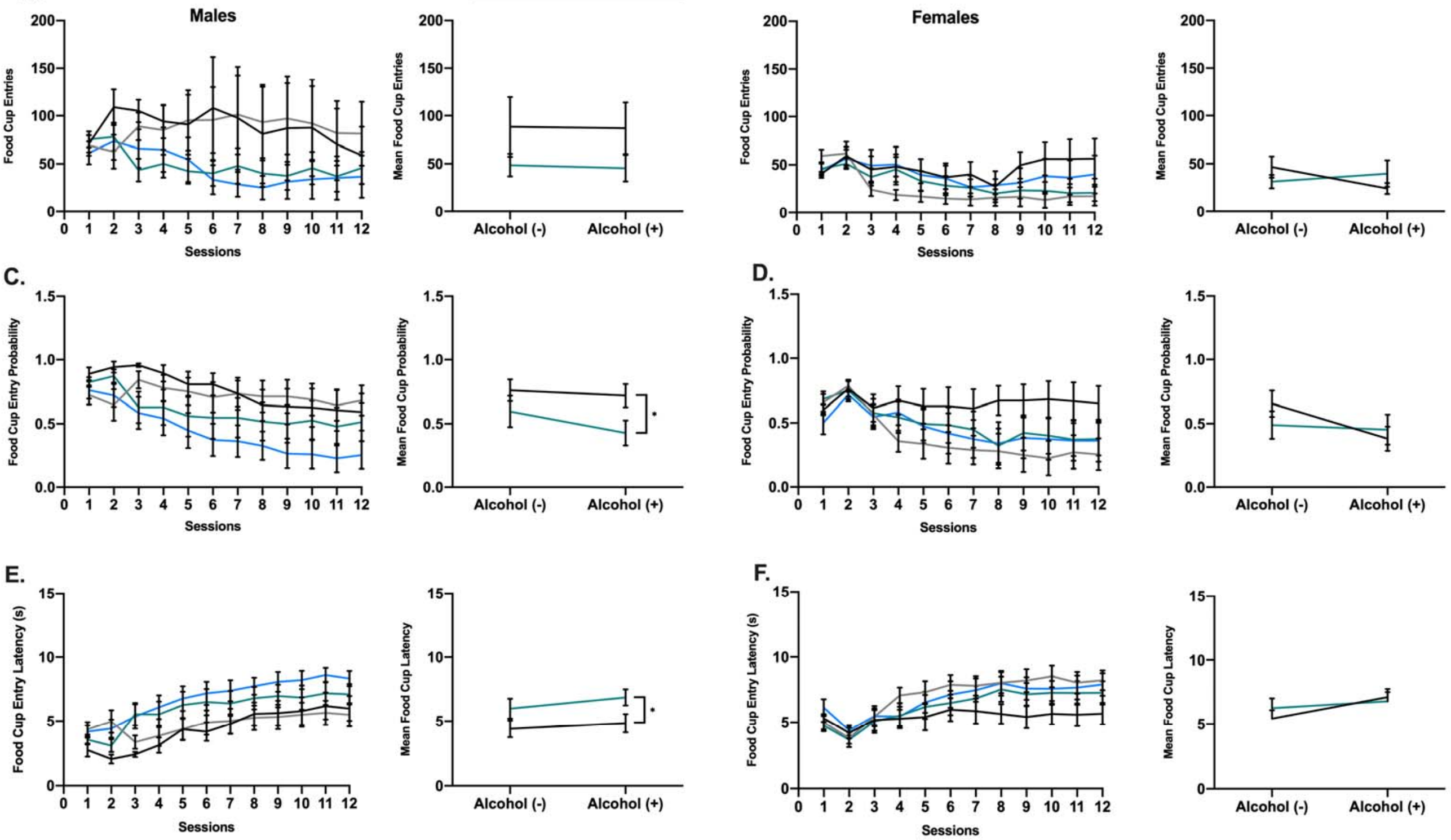


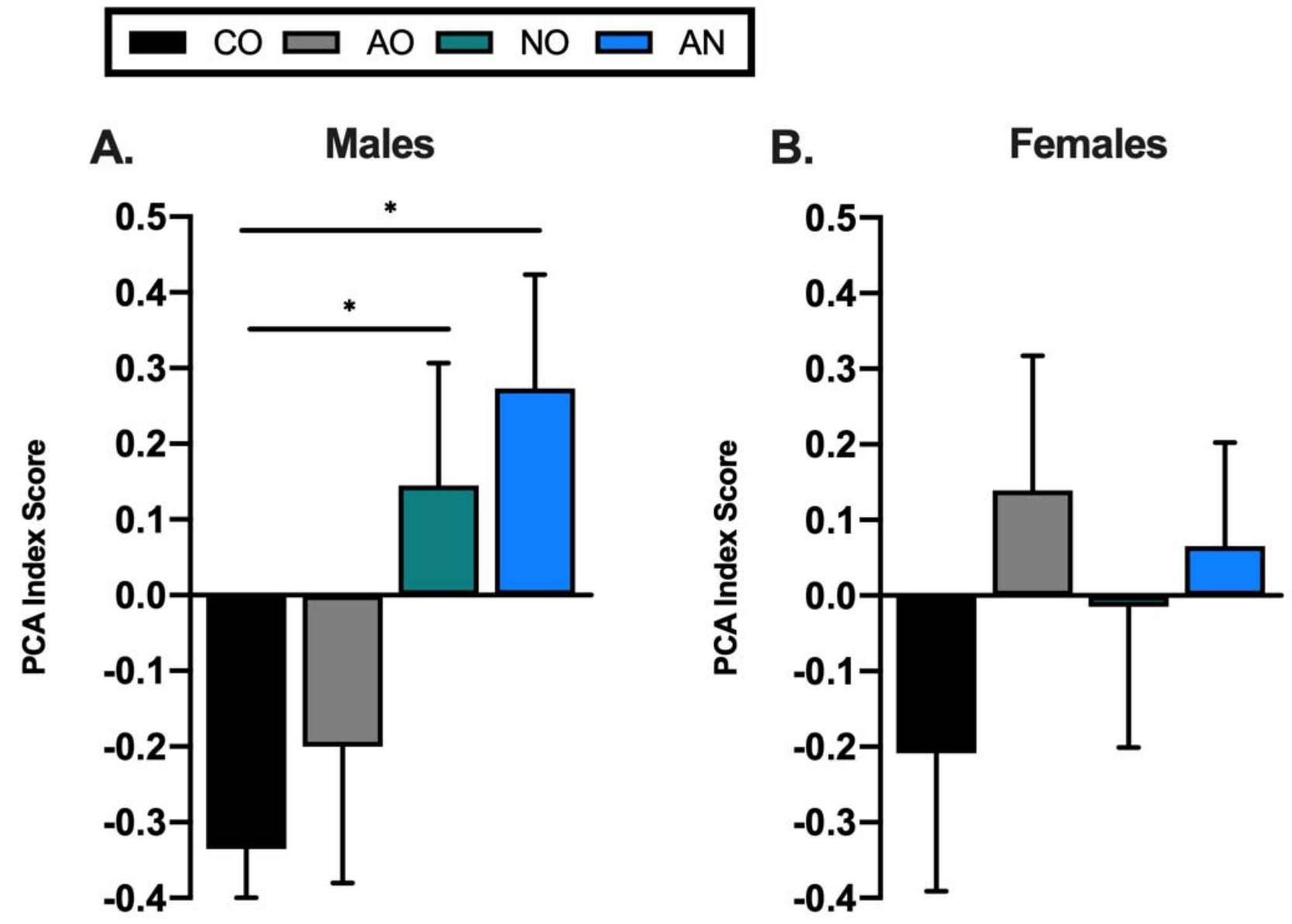




\section{Males}

$$
-\mathrm{CO}-\mathrm{AO}-\mathrm{NO}-\mathrm{AN}
$$

A.

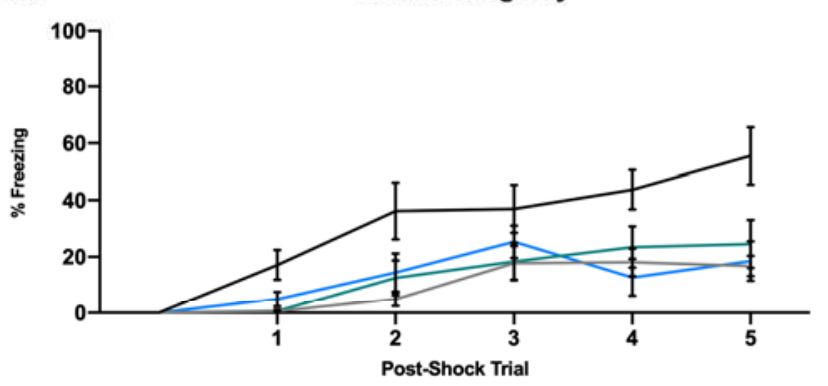

c.

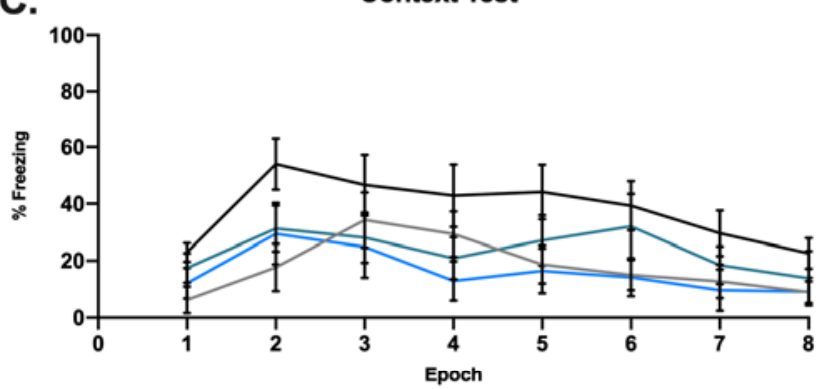

E.

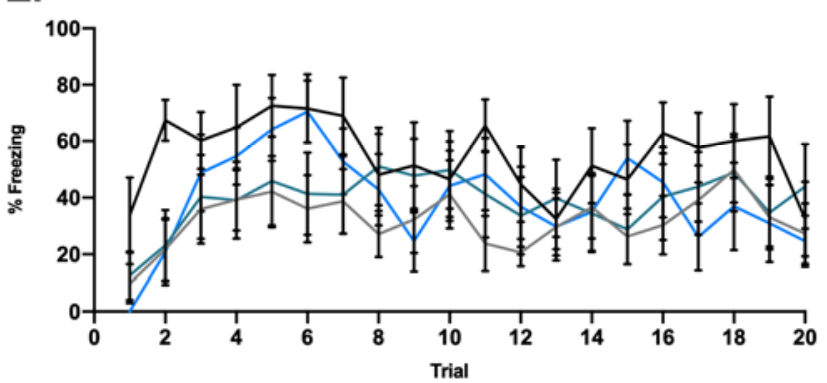

- Nicotine $(-)-$ Nicotine $(+)$
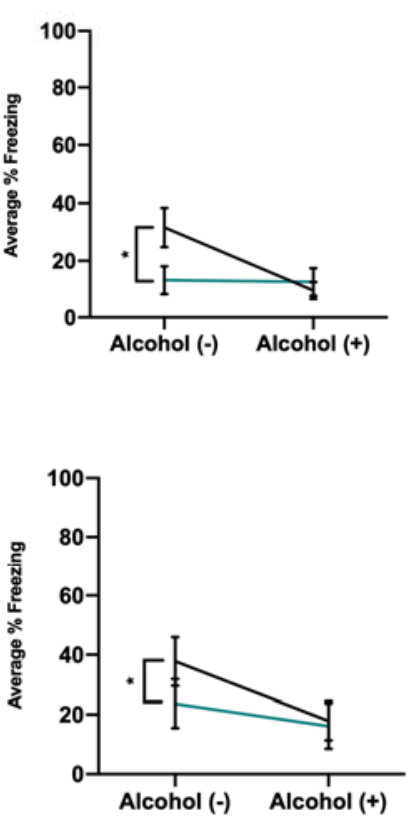

D.

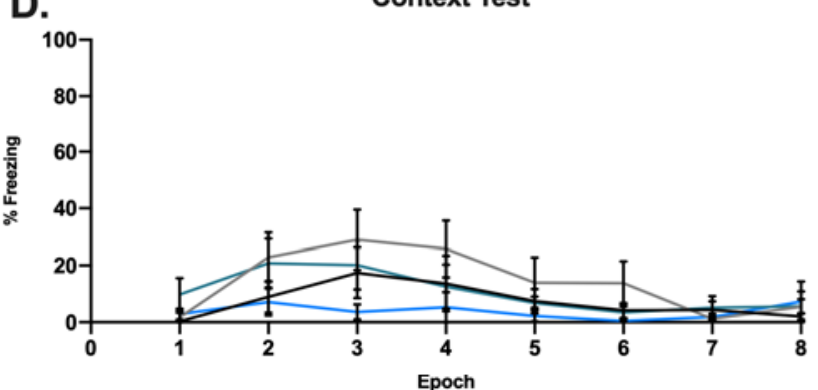

F.

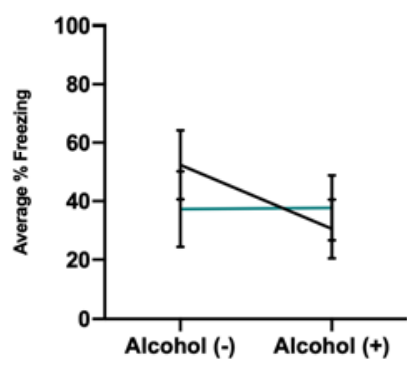

Females

B.
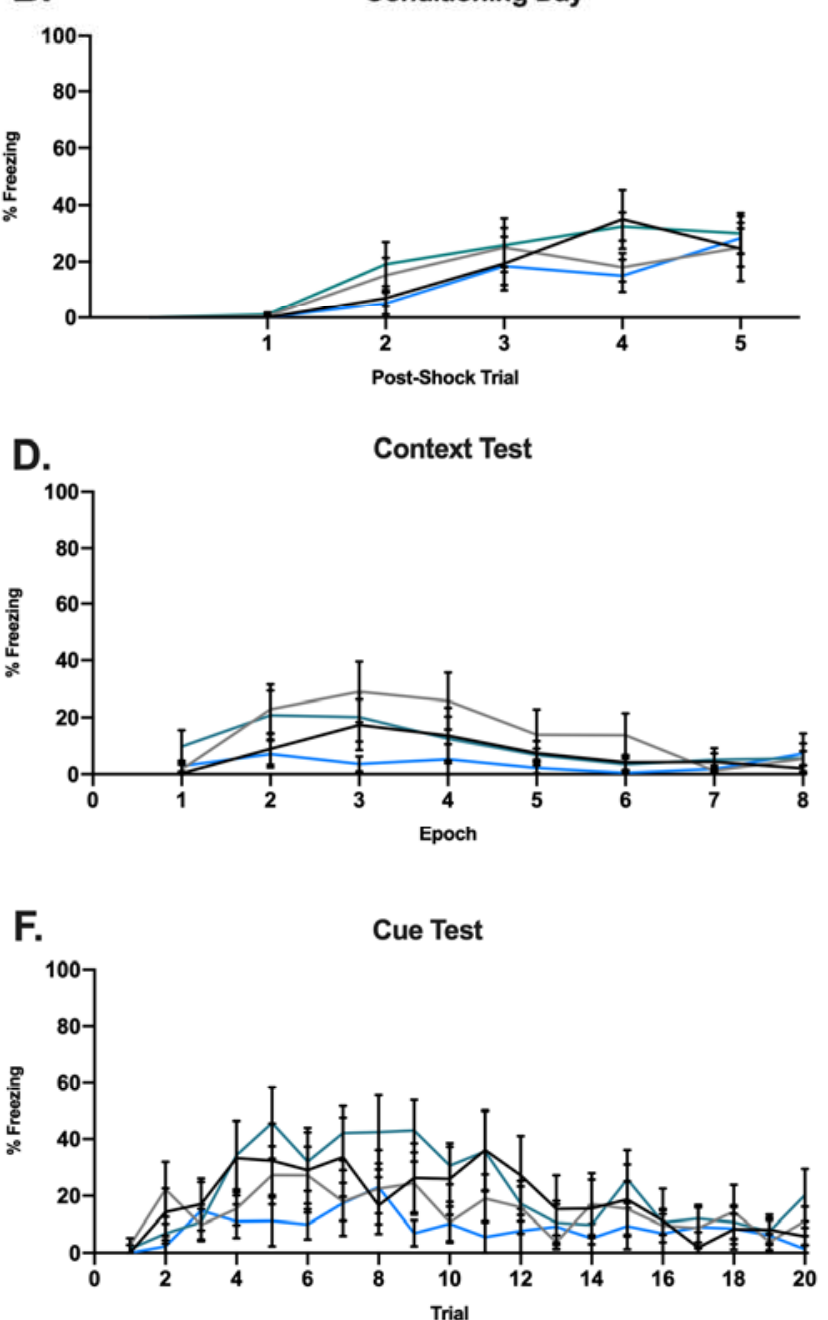
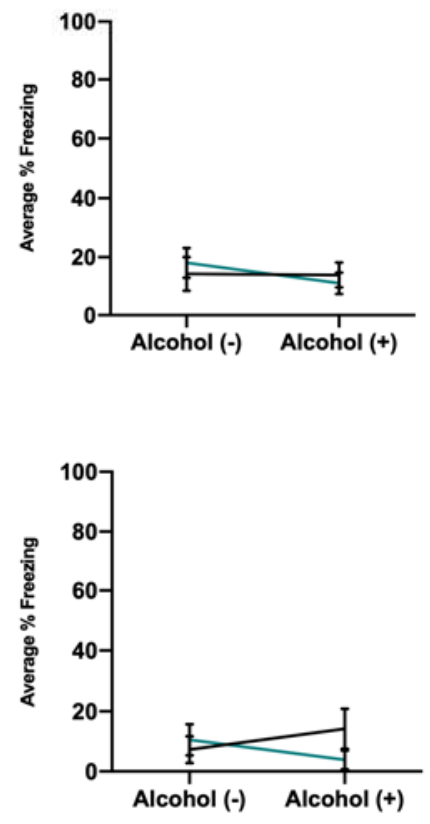

Alcohol (-) Alcohol (+)
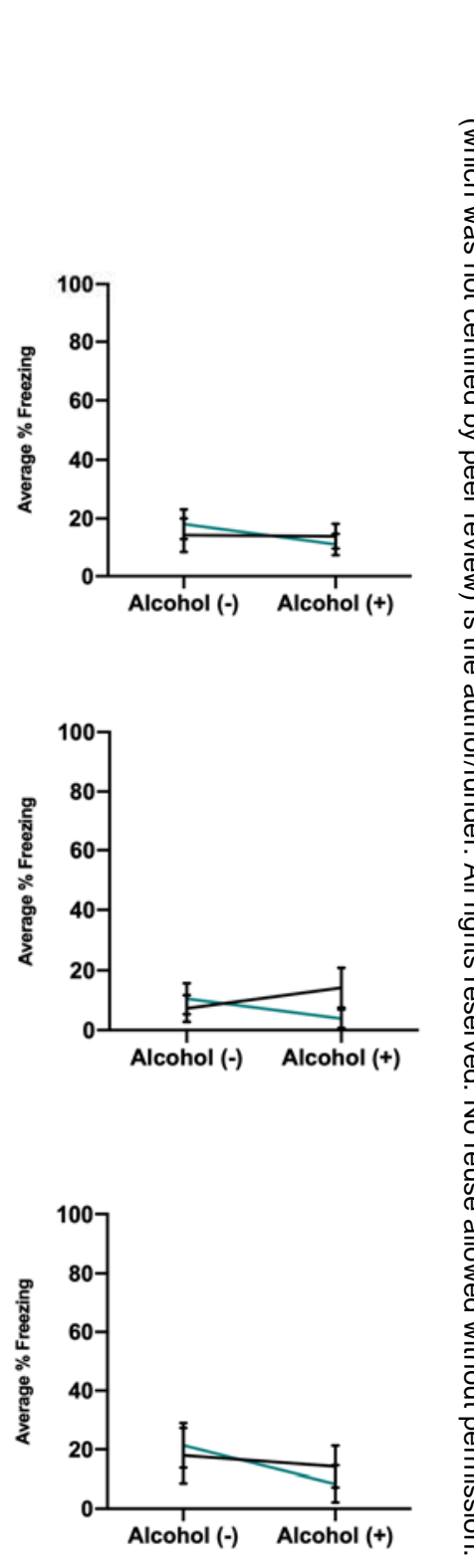